Regular Paper

\title{
Vector Magnetic Characteristic Analysis by Using Complex E\&S Model Taking Account of Stress Effect
}

\author{
Hayato ANDO*1, Yuichiro KAI ${ }^{* 1}$ and Masato ENOKIZONO*1
}

\begin{abstract}
This paper presents vector magnetic characteristic analysis by using the complex E\&S modelling taking account of stress effect. According to the past researches, we have evaluated the magnetic field strength $\boldsymbol{H}$ vector under controlled stresses and sinusoidal magnetic flux density waveforms. However, it is impossible to control the magnetic flux density $\boldsymbol{B}$ in actually magnetized motor cores. Therefore, it is necessary to know the relationships between $\boldsymbol{B}$ and $\boldsymbol{H}$-vector under the controlled stress and the non-controlled magnetic flux conditions. In this paper, we report the measured and calculated relationship between $\boldsymbol{B}$ - and $\boldsymbol{H}$-vector under stressed conditions and given sinusoidal voltage conditions. The trajectories and parameters of the $\boldsymbol{B}$ - and $\boldsymbol{H}$-vector under stress, calculated by using the StressComplex E\&S (SCE\&S) modeling, were agreed well with those of the measured $\boldsymbol{B}$ - and $\boldsymbol{H}$-vector.
\end{abstract}

Keywords: vector magnetic property, finite element method, stress effect.

(Received:24 July 2014)

\section{Introduction}

In recent years, it is required to improve efficiency and to decrease losses of motors because over $50 \%$ of the electric powers generated in Japan are consuming by motors. Therefore we should evaluate accurately magnetic properties of the electrical steel sheets utilizing in motors.

On the other hand, it is well known that the magnetic properties of the electrical steel sheets are generally deteriorated due to mechanical stress [1-3]. Therefore, it is necessary to develop new techniques for designing low loss and high efficiency motors. For these reasons, we have so far proposed the Stress-Complex E\&S (SCE\&S) modelling to evaluate accurately vector magnetic properties under stress in the electrical sheet sheets [1-3].

According to the past researches, we have evaluated the field strength changes due to stress under controlling the magnetic flux density $\boldsymbol{B}$ to be a sinusoidal waveform. However, practically, the magnetic flux density $\boldsymbol{B}$ is also changes due to stress as well as the field strength $\boldsymbol{H}$.

In this paper, to make clear the relationship between the magnetic flux density $\boldsymbol{B}$ and the field strength $\boldsymbol{H}$ under stressed conditions, a vector magnetic property measurement device is analyzed under sinusoidal voltage conditions by using the SCE\&S model and the calculated results are compared with the measured ones.

\section{Vector Magnetic Properties under Stress}

\subsection{Measurement system and specimen}

Correspondence: H. ANDO, Department of Electrical and Electronic Engineering, Faculty of Engineering, Oita University, Oita 870-1192, Japan email: v13e2002@oita-u.ac.jp

${ }^{* 1}$ Oita University

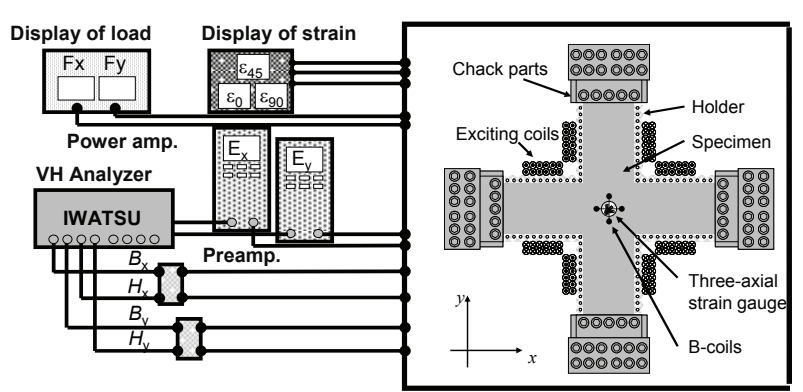

Fig. 1. Measurement system of vector magnetic property under stress.
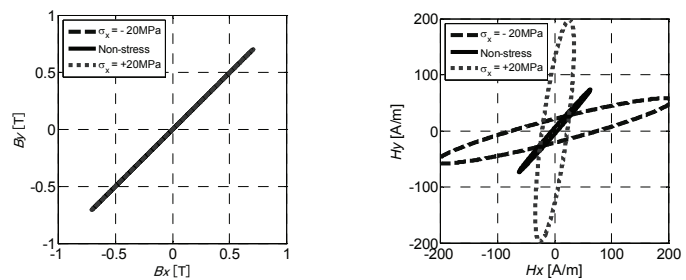

(a) Alternating magnetic flux condition
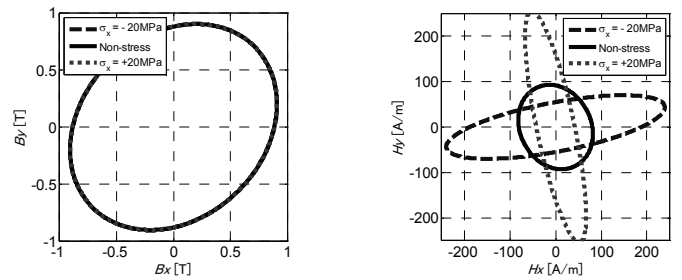

(b) Rotating magnetic flux condition

Fig. 2. The measured $\boldsymbol{B}$ and $\boldsymbol{H}$ trajectories under controlled alternating and rotating magnetic flux conditions.

Fig. 1 shows the vector magnetic property measurement system, which can apply stress. Fig. 2 shows the measured $\boldsymbol{B}$ and $\boldsymbol{H}$ trajectories under the controlled alternating and rotating magnetic flux conditions. The tensile and compressive stresses are applied to the specimen along the rolling direction. The magnetic flux density vector with and without stress is controlled as shown in Fig. 2. The amplitude and inclination angle 


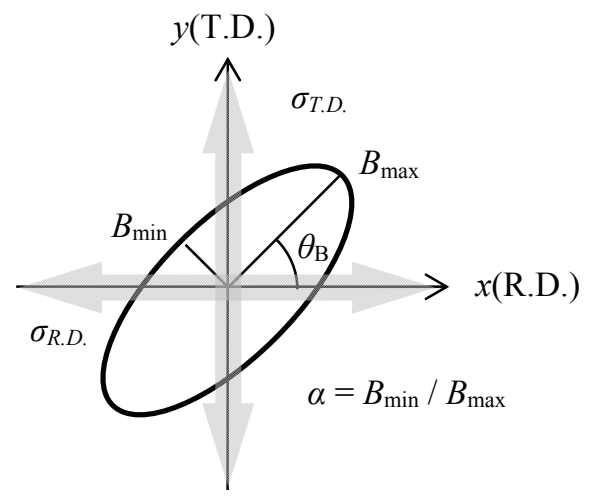

Fig. 3. Definition of the magnetic flux conditions and stress for SCE\&S modelling.

of the $\boldsymbol{H}$ trajectories were varied with the stress. The value of $H_{\mathrm{y}}$ in $y$ direction increased with the tensile stress at $\sigma_{\mathrm{x}}=20 \mathrm{MPa}$. The value of $H_{\mathrm{x}}$ in $x$ direction increased with the compressive stress at $\sigma_{\mathrm{x}}=-20 \mathrm{MPa}$. In this paper, we try to examine effect of the stress on not only the magnetic field strength vector but also the magnetic flux density vector under non-controlled magnetic flux conditions.

\subsection{Stress-Complex E\&S modeling}

Fig. 3 shows parameters used in definition of the SCE\&S modelling. The SCE\&S modelling can be defined with five parameters: $B_{\max }, \theta_{\mathrm{B}}, \alpha, \sigma_{\text {R.D. }}$, and $\sigma_{\text {T.D. }}$. $B_{\max }$ is the maximum magnetic flux density, $\theta_{\mathrm{B}}$ is the angle between the rolling direction (R.D) of an electrical steel sheet and the direction of $B_{\max } \alpha$ is the ratio of the minimum magnetic flux density and $B_{\max }$. $\sigma_{\text {R.D. }}$ is the stresses in R.D. and $\sigma_{\text {T.D. }}$ is the stresses in the transverse direction (T.D.). The SCE\&S modelling can be written as

$$
\begin{aligned}
& \dot{H}_{x}=\bar{v}_{x r}\left(B_{\max }, \alpha, \theta_{B}, \sigma_{R . D .}, \sigma_{T . D .}\right) \dot{B}_{x} \\
& +j \omega \bar{v}_{x i}\left(B_{\max }, \alpha, \theta_{B}, \sigma_{R . D .}, \sigma_{T . D .}\right) \dot{B}_{x} \\
& \dot{H}_{y}=\bar{v}_{y r}\left(B_{\max }, \alpha, \theta_{B}, \sigma_{R . D .}, \sigma_{T . D .}\right) \dot{B}_{y} \\
& +j \omega \bar{v}_{y i}\left(B_{\max }, \alpha, \theta_{B}, \sigma_{R . D .}, \sigma_{T . D .}\right) \dot{B}_{y}
\end{aligned}
$$

where $B_{\mathrm{x}}$ and $B_{\mathrm{y}}$ are the components of the magnetic flux density, $H_{\mathrm{x}}$ and $H_{\mathrm{y}}$ are the components of the magnetic field strength, $v_{\mathrm{xr}}$, $v_{\mathrm{yr}}$ are the magnetic reluctivity coefficients, and $v_{\mathrm{xi}}, v_{\mathrm{yi}}$ are the magnetic hysteresis coefficients. The variables with upper point mean complex variables and the over bar means effective variables.

The magnetic reluctivity coefficients and the magnetic hysteresis coefficients in $x$ - and $y$-direction can be calculated as

$$
\left\{\begin{array}{l}
\bar{v}_{x r}=\frac{\left|\dot{H}_{x}\right|}{\left|\dot{B}_{x}\right|} \cos \left(\theta_{B H_{x}}\right), \bar{\nu}_{x i}=\frac{\left|\dot{H}_{x}\right|}{\omega\left|\dot{B}_{x}\right|} \sin \left(\theta_{B H_{x}}\right) \\
\bar{\nu}_{y r}=\frac{\left|\dot{H}_{y}\right|}{\left|\dot{B}_{y}\right|} \cos \left(\theta_{B H_{y}}\right), \bar{v}_{y i}=\frac{\left|\dot{H}_{y}\right|}{\omega\left|\dot{B}_{y}\right|} \sin \left(\theta_{B H_{y}}\right)
\end{array}\right.
$$

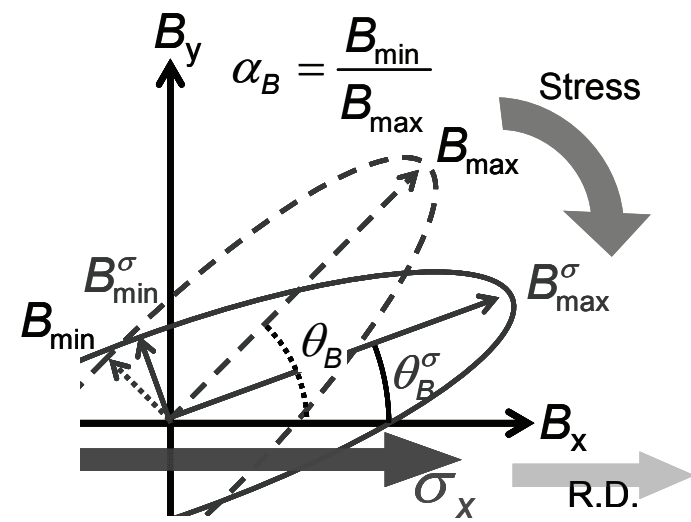

(a) $\boldsymbol{B}$ trajectories

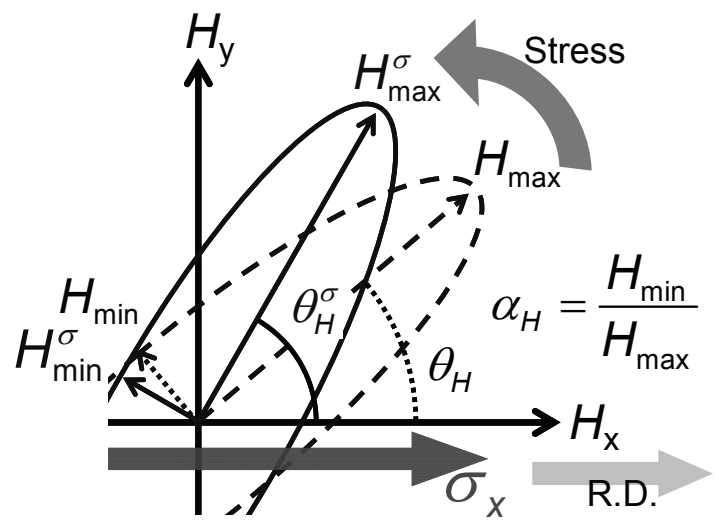

(b) $\boldsymbol{H}$ trajectories

Fig. 4. Parameters of the $\boldsymbol{B}$ - and $\boldsymbol{H}$-trajectories with and without stress.

where $\theta_{\mathrm{BH}}$ is the phase difference between the $\boldsymbol{B}$ - and $\boldsymbol{H}$ waveforms in $x$ - and $y$-direction.

\subsection{Parameters of the $\boldsymbol{B}$ - and $\boldsymbol{H}$-trajectories with and without stress}

Fig. 4 shows the parameters of the $\boldsymbol{B}$ - and $\boldsymbol{H}$ trajectories with and without stress. When the magnetic flux density vector is controlled, the parameters of the magnetic flux density with and without stress are defined as $B_{\max }, \alpha_{\mathrm{B}}$, and $\theta_{\mathrm{B}}$. In this case, the magnetic flux density is changed by applying stress because the magnetic flux density waveform is not controlled to be sinusoidal. New parameters of the magnetic flux density with the stress are defined as $B^{\sigma}{ }_{\max }, \alpha_{\mathrm{B}}^{\sigma}$, and $\theta^{\sigma}{ }_{\mathrm{B}}$ as shown in Fig. 4(a).

On the other hand, the parameters of the field strength without stress are defined as $H_{\max }, \alpha_{\mathrm{H}}$, and $\theta_{\mathrm{H}}$. Here, 
$H_{\max }$ is the maximum field strength, $\alpha_{\mathrm{H}}$ is the angle between the rolling direction and the direction of $H_{\max }$, and $\theta_{\mathrm{H}}$ is the ratio of $H_{\min }$ and $H_{\max }$. In addition, the parameters of the magnetic flux density with the stress are defined as $H^{\sigma}{ }_{\max }, \alpha_{\mathrm{H}}^{\sigma}$, and $\theta_{\mathrm{H}}^{\sigma}$ as shown in Fig. 4(b).

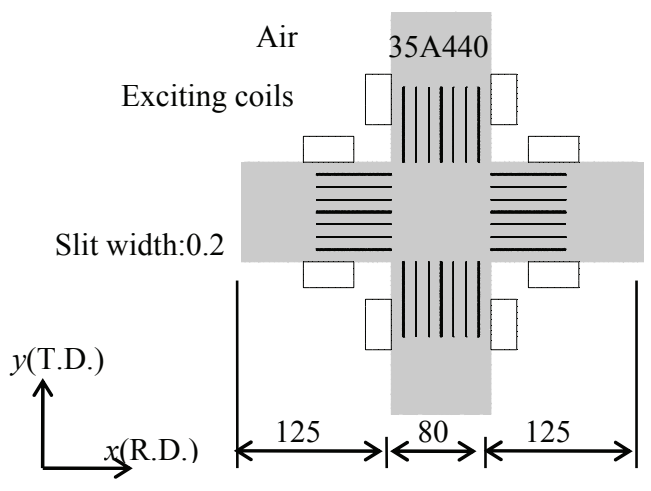

Fig. 5. Analyzed model

Table 1 Conditions used in the analysis

\begin{tabular}{|c|c|c|}
\hline \multicolumn{2}{|c|}{ Electrical steel sheet } & $35 \mathrm{~A} 440$ \\
\hline \multicolumn{2}{|c|}{ Stress condition $\sigma_{\mathrm{x}}$} & $-20 \sim+30(10$ steps $) \mathrm{MPa}$ \\
\hline \multirow{2}{*}{ Coil } & Number of winding & 100 turns $/$ coil \\
\cline { 2 - 3 } & Resistance & $1.0 \Omega$ \\
\hline
\end{tabular}

The $\boldsymbol{B}$ - and $\boldsymbol{H}$-trajectories change locally due to residual stress as a practical phenomenon. Therefore, the parameters of $\boldsymbol{B}$ and $\boldsymbol{H}$ change with changing stress in the same way.

\section{Analyzed model and conditions}

\subsection{Analyzed model}

Fig. 5 shows the analyzed model. The shape and the dimension of the cross-shaped specimen are similar to ones of the measurement device. We evaluated the magnetic property at the center of the specimen for varies stressed conditions. The tensile and compressive stresses are applied to specimen along the rolling direction.

\subsection{Conditions used in the analysis}

Table 1 shows the conditions used in the analysis. The non-oriented electrical steel sheet, which is named 35A440 in Japanese Industry Standards, is used as a specimen. The cross-shaped specimen has few slits in order to apply stress uniformly in the specimen. The amplitude of the stress is changed from $-20 \mathrm{MPa}$ to 30 $\mathrm{MPa}$. After assuming a stress condition, the specimen is magnetized in $x$ - and $y$-direction by applying the terminal voltage in the simulation.

Fig. 6 shows the flowchart of setting the exciting conditions. At first, the exciting voltage waveform without stress is controlled to be an objected alternating or rotating magnetic flux condition as shown in Fig. 2. The exciting voltage is modified with the method of the Rosen-Brock until the calculated results are corresponded to be the objected flux condition. Next, the tensile and compressive stress is applied to the specimen as shown in Table 1, and the specimen is magnetized by using the controlled exciting voltage under the alternating magnetic flux conditions without stress. After that, the $\boldsymbol{B}$ - and $\boldsymbol{H}$-trajectories with and without stress are evaluated within the central $20 \mathrm{~mm} \times 20 \mathrm{~mm}$ region as shown in Fig 5.

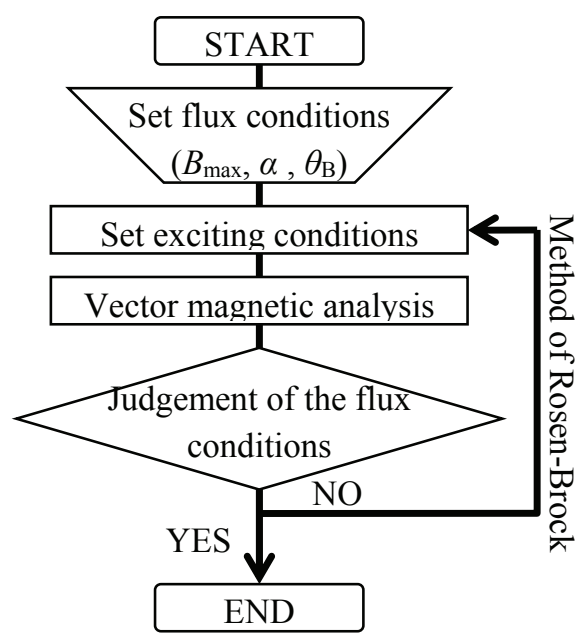

Fig. 6. Flowchart of setting the exciting condition

\section{Results and discussions}

\subsection{Alternating magnetic flux condition}

Fig. 7 shows the calculated and measured $\boldsymbol{B}$ trajectories under the alternating magnetic flux condition. The $\boldsymbol{B}$-trajectories were changed by applying the stress. The calculated and measured $\boldsymbol{B}$-trajectories were in a good agreement in tendency. In particular, the inclination angles of the calculated and measured $\boldsymbol{B}$ trajectories decreased with the tensile stress and increased with the compressive stress, because the permeability in $x$ - and $y$-direction changes due to the stress. In particular, the magnetic permeability along the direction parallel to the tensile stress increased and the one along the transverse direction parallel to the compressive stress increased.

Fig. 8 shows the calculated and measured parameters of $\boldsymbol{B}$-trajectories under the alternating magnetic flux condition. The value of $B_{\max }$ and $\theta_{\mathrm{B}}$ decreased with increasing the tensile stress and increased with increasing the compressive stress. On the other hand, the values of $\alpha_{\mathrm{B}}$ slightly changed with increasing the stress because the value of $\alpha_{\mathrm{B}}$ under the alternating magnetic flux condition was usually very small. 


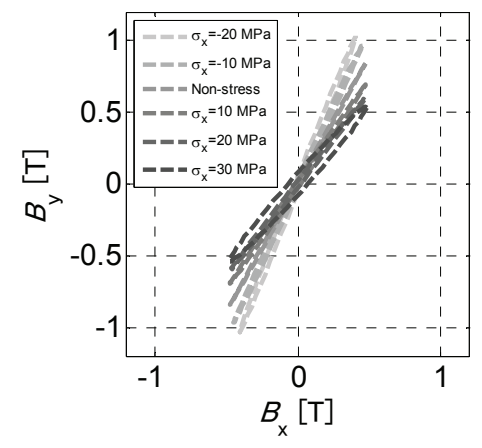

(a) Calculated $\boldsymbol{B}$-trajectories

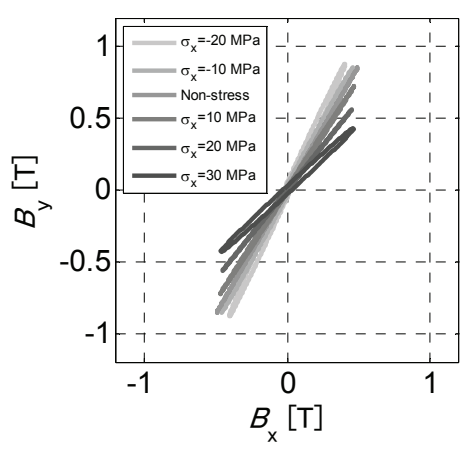

(b) Measured $\boldsymbol{B}$-trajectories

Fig. 7. Calculated and measured $\boldsymbol{B}$-trajectories depending on stress under the alternating magnetic flux condition.

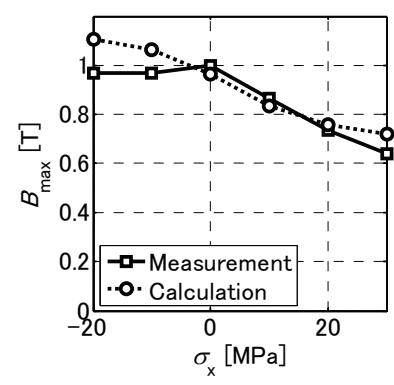

(a) $B_{\max }$ and $B^{\sigma}{ }_{\max }$

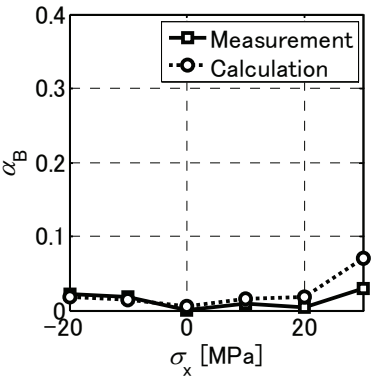

(b) $\alpha_{\mathrm{B}}$ and $\alpha^{\sigma}{ }_{\mathrm{B}}$

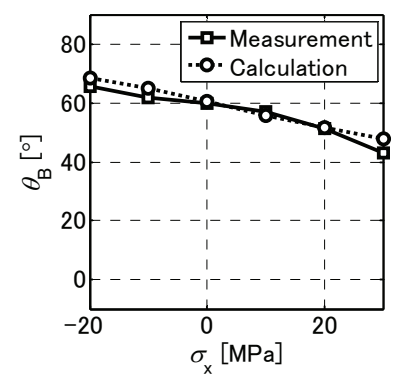

(c) $\theta_{\mathrm{B}}$ and $\theta^{\sigma_{\mathrm{B}}}$

Fig. 8. Calculated and measured parameters of $\boldsymbol{B}$-trajectories depending on stress under the alternating magnetic flux condition.

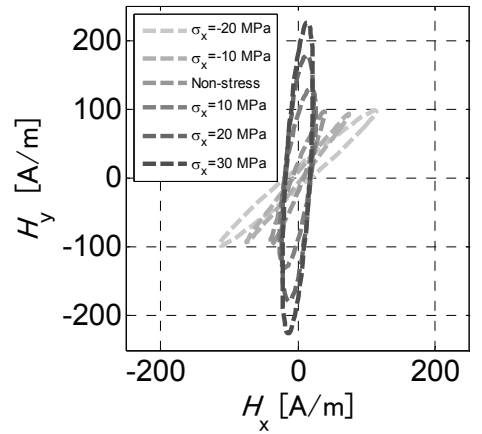

(a) Calculated $\boldsymbol{H}$ trajectories

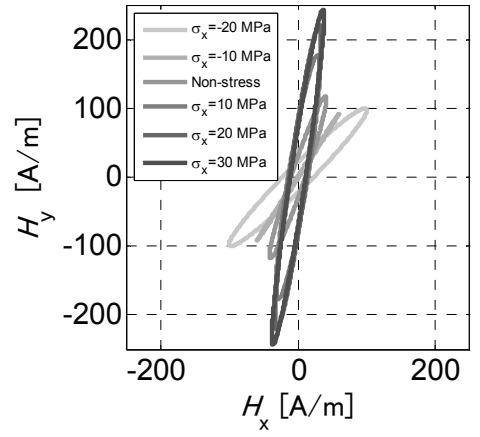

(b) Measured $\boldsymbol{H}$ trajectories

Fig. 9. Calculated and measured $\boldsymbol{H}$-trajectories depending on stress under the alternating magnetic flux condition.

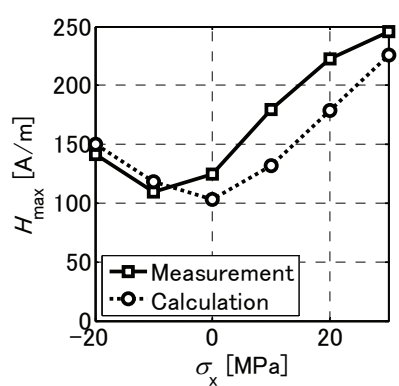

(a) $H_{\max }$ and $H^{\sigma}{ }_{\max }$

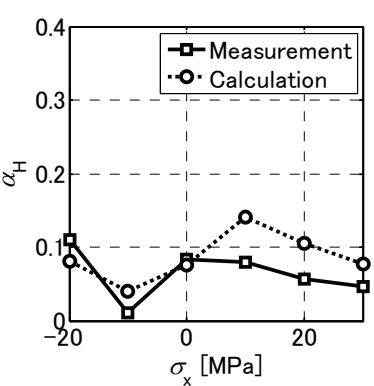

(b) $\alpha_{\mathrm{H}}$ and $\alpha_{\mathrm{H}}^{\sigma}$

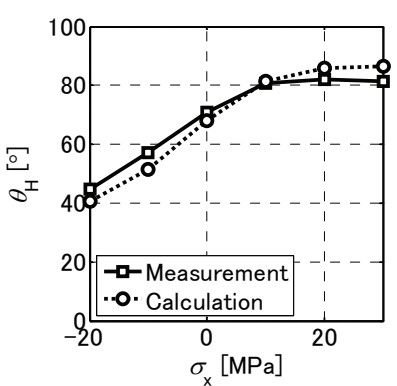

(c) $\theta_{\mathrm{H}}$ and $\theta_{\mathrm{H}}^{5}$

Fig. 10. Calculated and measured parameters of $\boldsymbol{H}$-rajectories depending on stress under the alternating magnetic flux condition. 


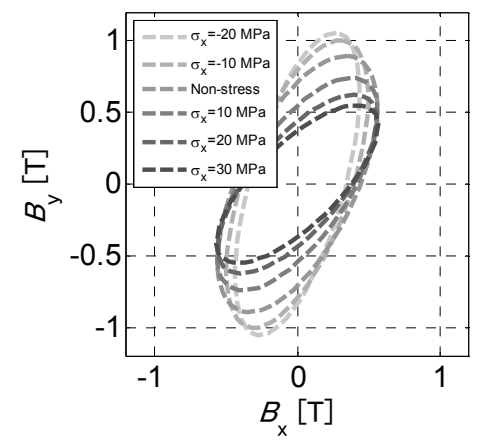

(a) Calculated $\boldsymbol{B}$-trajectories

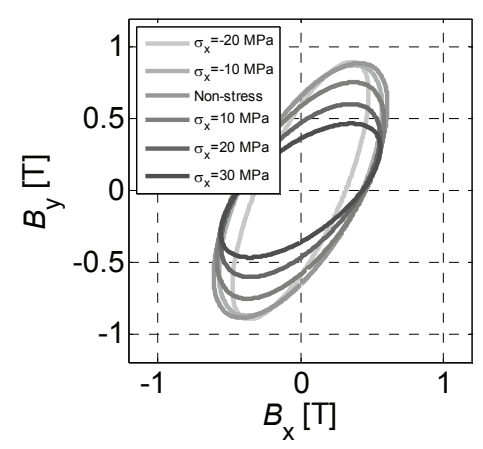

(b) Measured $\boldsymbol{B}$-trajectories

Fig. 11. Calculated and measured $\boldsymbol{B}$-trajectories depending on stress under the rotating magnetic flux condition.

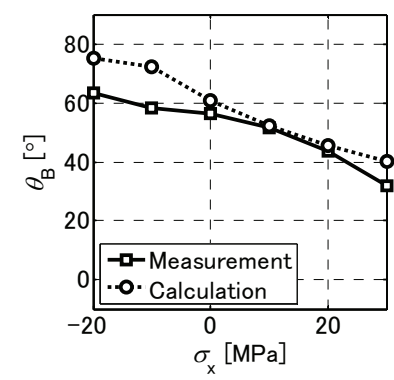

(a) $B_{\max }$ and $B_{\text {max }}^{\sigma}$

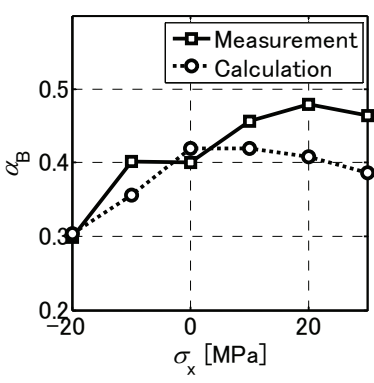

(b) $\alpha_{\mathrm{B}}$ and $\alpha^{\sigma}{ }_{\mathrm{B}}$

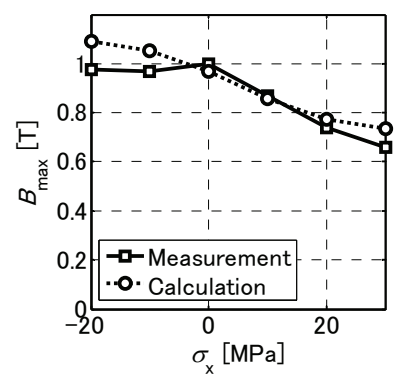

(c) $\theta_{\mathrm{B}}$ and $\theta^{\sigma_{\mathrm{B}}}$

Fig. 12. Calculated and measured parameters of $\boldsymbol{B}$-trajectories depending on stress under the rotating magnetic flux condition.

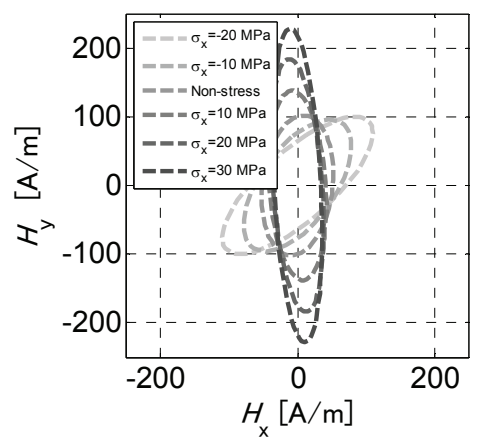

(a) Calculated $\boldsymbol{H}$ trajectories

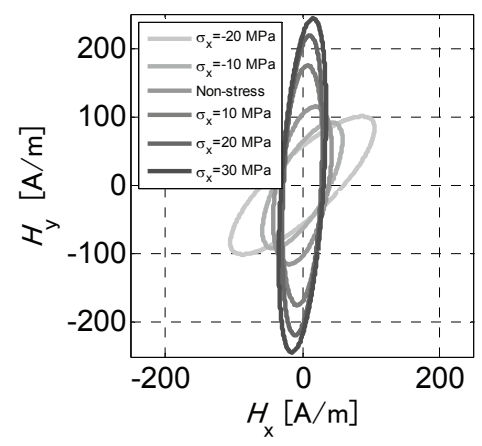

(b) Measured $\boldsymbol{H}$ trajectories

Fig. 13. Calculated and measured $\boldsymbol{H}$-trajectories depending on stress under the rotating magnetic flux condition.

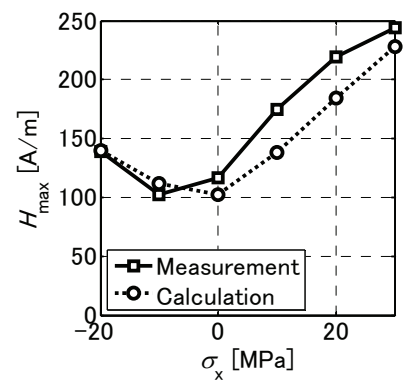

(a) $H_{\max }$ and $H^{\sigma}{ }_{\max }$

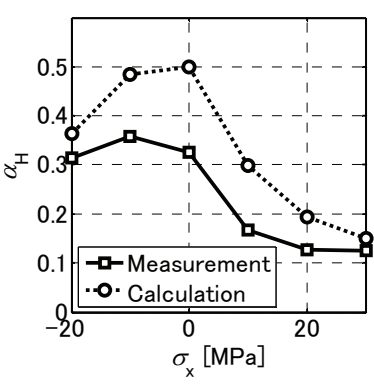

(b) $\alpha_{\mathrm{H}}$ and $\alpha_{\mathrm{H}}^{\sigma}$

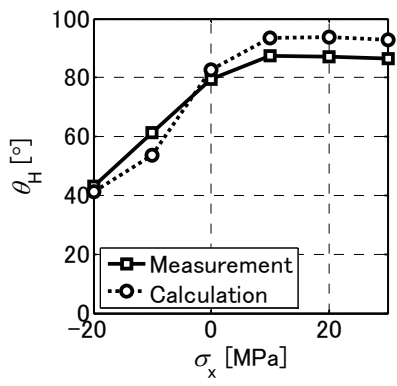

(c) $\theta_{\mathrm{H}}$ and $\theta_{\mathrm{H}}$

Fig. 14. Calculated and measured parameters of $\boldsymbol{H}$-trajectories depending on stress under the rotating magnetic flux condition. 
Fig. 9 shows the calculated and measured $\boldsymbol{H}$ trajectories under the alternating magnetic flux condition. The inclination angles of the calculated and measured $\boldsymbol{H}$ - trajectories increased with the tensile stress and decreased with the compressive stress. The change of the calculated and measured $\boldsymbol{H}$-trajectories became larger than those of $\boldsymbol{B}$-trajectories. It was made clear that the magnetic field strength was influenced strongly by stress in comparison with the magnetic flux density.

Fig. 10 shows the calculated and measured parameters of the $\boldsymbol{H}$-trajectories under the alternating magnetic flux condition. The value of $H_{\max }$ increased with increasing the tensile and compressive stress. The value of the $\theta_{\mathrm{H}}$ increased with increasing the tensile stress and decreased with increasing the compressive stress. In addition, the value of $\alpha_{\mathrm{H}}$ differed for each stress because the rotating magnetic field was also generated due to the stress magnetic anisotropy.

From these results, it was clarified that the calculated and measured $\boldsymbol{B}$ - and $\boldsymbol{H}$ - trajectories and the parameters under the alternating magnetic flux condition were in good agreements in tendency.

\subsection{Rotating magnetic flux condition}

Fig. 11 shows the calculated and measured $\boldsymbol{B}$ trajectories under the rotating magnetic flux condition. The shape and inclination angle of the calculated and measured $\boldsymbol{B}$-trajectories differed in the case with and without the stress. The inclination angle of the calculated and measured $\boldsymbol{B}$ - trajectories decreased with the tensile stress and increased with the compressive stress.

Fig. 12 shows the calculated and measured parameters of $\boldsymbol{B}$-trajectories under the rotating magnetic flux condition. The value of $B_{\max }$ and $\theta_{\mathrm{B}}$ decreased with increasing the tensile stress and increased with increasing the compressive stress. On the other hand, the value of $\alpha_{\mathrm{B}}$ increased with increasing the tensile stress and decreased with increasing the compressive stress.

Fig. 13 shows the calculated and measured $\boldsymbol{H}$ trajectories under the rotating magnetic flux condition. The inclination of the calculated and measured $\boldsymbol{H}$ trajectories increased with the tensile stress and decreased with the compressive stress.

Fig. 14 shows the calculated and measured parameters of $\boldsymbol{H}$-trajectories under the rotating magnetic flux condition. The value of $H_{\max }$ increased with increasing both of the tensile and compressive stresses. The value of the $\theta_{\mathrm{H}}$ increased with increasing the tensile stress and decreased with increasing the compressive stress. On the other hand, the calculated and measured $\alpha_{\mathrm{H}}$ showed similar tendency for the stress.

As a result, it was shown that the calculated and measured $\boldsymbol{B}$ - and $\boldsymbol{H}$-trajectories and the parameters under the rotating magnetic flux condition were in good agreements, however, differences of the calculated and measured parameters became larger by increasing stress. The cause can be considered as harmonic components of the magnetic field strength waveforms, which are neglected in the SCE\&S modeling.

\section{Conclusion}

The effect of stress on the magnetic flux density and magnetic field strength vector under the both alternating and rotating magnetic flux conditions was measured and calculated by using the SCE\&S modelling. The SCE\&S vector characteristic analysis was carried out for the cross-shaped specimen placed in the measurement device. The magnetic flux density vector and magnetic field strength vector were changed depending on the applied stress. In addition, it was made clear that the calculated and measured $\boldsymbol{B}$ - and $\boldsymbol{H}$-trajectories and the parameters under the both alternating and rotating magnetic flux conditions were in good agreements in tendency. As a result, it can be said that it is possible to use the SCE\&S modelling as a design toll of motors in taking account of the stress effect. However, the differences of the calculated and measured magnetic field strength vector under the rotating magnetic flux condition remained as an issue. In future works, more accuracy improvement of the modeling is needed in order to consider distortions of the magnetic field strength waveforms under the stress conditions.

\section{References}

[1] Z. Gmyrek, M. Strakowska, B. Wiecek, "A method of local magnetic loss determination in punched ferromagnetic strips," Journal of Magnetism and Magnetic Materials, 355, pp.282-288, 2014.

[2] A. J. Moses and H. Rahatizadeh, "Effects of stress on iron loss and flux distribution of an induction motor stator core," IEEE Transaction on Magnetics, Vol. 25, No. 5, pp. 4003-4005, 1984.

[3] Y. Kai, Y. Tsuchida, T. Todaka, M. Enokizono, "Influence of stress on vector magnetic property under alternating magnetic flux conditions", IEEE Transactions on Magnetics, Vol. 47, No. 10, pp.4344-4347, 2011.

[4] M. Enokizono, and N. Soda, "Direct magnetic loss analysis by FEM considering vector magnetic property," IEE Trans. Magn., Vol.34, pp.188-195, 1998.

[5] S. Zeze, Y. Kai, T. Todaka, and M. Enokizono, "Vector magnetic Characteristic Analysis of a PM Motor Considering Residual Stress Distribution With Complex - Approximated Material Modeling," IEEE Transactions on magnetics, Vol. 48, NO. 11, pp.3352-3355, 2012.

[6] Y. Kai, T. Todaka, and M. Enokizono, "Evaluation of local residual stress distribution of stator core in rotating machine," IEEJ Trans. FM,, vol. 131, no.5, pp.389-394, 2011. 